03

\title{
Результаты испытаний активной системы снижения вибрационных сил и пульсаций давления
}

\author{
(C) А.В. Кирюхин ${ }^{1,2}$, О.О. Мильман ${ }^{1,2}$, А.В. Птахин ${ }^{2}$, \\ Л.Н. Сережкин ${ }^{1}$ \\ ${ }^{1}$ Калужский государственный университет им. К.Э. Циолковского, \\ Калуга, Россия \\ ${ }^{2}$ Научно-производственное внедренческое предприятие „Турбокон“, \\ Калуга, Россия \\ E-mail: turbocon@kaluga.ru
}

Поступило в Редакцию 28 июня 2018 г.

Отмечается отсутствие в литературе данных по снижению передачи вибрации и пульсации давления через компенсаторы трубопроводов с жидкостью активными методами. Экспериментально исследован опытный образец активной системы широкополосного гашения вибрационных сил и пульсаций давления, определяющих передачу вибрации через компенсаторы трубопроводов с жидкостью. Приведены результаты испытаний активной системы с электромагнитным вибратором в качестве источника компенсирующей силы, пьезокерамическим излучателем в качестве источника компенсирующей пульсации давления и цифровым устройством управления. Получено снижение сил и пульсаций давления до $18 \mathrm{~dB}$ в полосе частот от 10 до $246 \mathrm{~Hz}$.

DOI: 10.21883 /PJTF.2018.24.47028.17443

Для снижения передачи вибрации в трубопроводах с жидкостью применяют гасители пульсаций давления и виброизолирующие вставки (компенсаторы) [1,2]. Анализ литературы показывает, что, несмотря на имеющееся большое количество работ по активному гашению вибраций, отсутствуют публикации, посвященные вопросу снижения передачи вибрации через компенсаторы трубопроводов с жидкостью, в том числе активными методами. Отсутствуют соответствующие физические и математические модели и методы расчета. Исследования авторов [2] показали, что из-за взаимодействия вибрации структуры компенсатора и пульсаций рабочей среды в нем с ростом частоты деформирования передача вибрации через компенсатор может возрастать на несколько 
порядков. Это осложняет создание конструкций компенсаторов с низкой передачей вибрации и применение активных методов. Отмеченная проблема важна в транспортном и энергетическом машиностроении.

Передачу вибрации через компенсаторы можно уменьшать снижением передаваемых вибрационных сил, уменьшая структурную жесткость упругих элементов компенсатора. Можно уменьшать пульсации давления, которые порождаются работой установки и вибрацией фланцев компенсатора. На практике эффективность пассивных методов снижения вибраций и пульсаций ограничена прочностью, габаритами и параметрами рабочих процессов в установках. Дальнейшее снижение передачи вибрации может быть получено при помощи активных систем (АС) [3].

Активные системы создают компенсирующие вибрационные силы, в частности действующие на присоединенные к установке конструкции в противофазе к силам, действующим от установки. Аналогично действуют АС подавления пульсаций давления в трубопроводе [3]. АС могут работать в узких или широких полосах частот. В первом случае они гасят дискретные компоненты в спектрах вибраций, во втором снижают случайные колебания в полосе частот. В настоящей работе приведены результаты испытаний опытной широкополосной двухканальной $\mathrm{AC}$ c электромагнитным вибратором в качестве источника компенсирующей силы, пьезокерамическим излучателем в качестве источника компенсирующей пульсации давления и цифровым устройством управления.

Схема АС и стенда для компенсации динамической силы и пульсации давления в трубопроводе приведена на рис. 1. Стенд состоит из металлической плиты 1 , установленной на датчиках вибрационной силы 2, закрепленных на фундаменте 3, трубы 4, заполненной водой. Внутренний диаметр трубы $100 \mathrm{~mm}$, высота $1.25 \mathrm{~m}$. Поршень 5 возбуждается электродинамическим вибратором $V_{p}$ с усилителем мощности $\mathrm{PA}_{p}$. Он создает пульсации давления $P$ в воде, измеряемые гидрофонами 6. Действуя на верхнюю и нижнюю крышки трубы 4, пульсации давления $P$ создают динамическую силу $F_{p}$ воздействия пульсаций в трубе на плиту 1. Канал структурного возбуждения вибрации состоит из электродинамического вибратора $V_{v}$ с усилителем мощности $\mathrm{PA}_{v}$. Он создает вибрационную силу $F_{v}$, действующую на плиту 1 . Сигналы возбуждения на усилители мощности подаются от генератора сигналов SG в виде широкополосного случайного сигнала (белого шума) либо синусоидального сигнала заданной частоты. Сила $F_{f}$ действия вибрации плиты 1 на фундамент 3 измеряется датчиками силы 2.

Письма в ЖТФ, 2018, том 44, вып. 24 


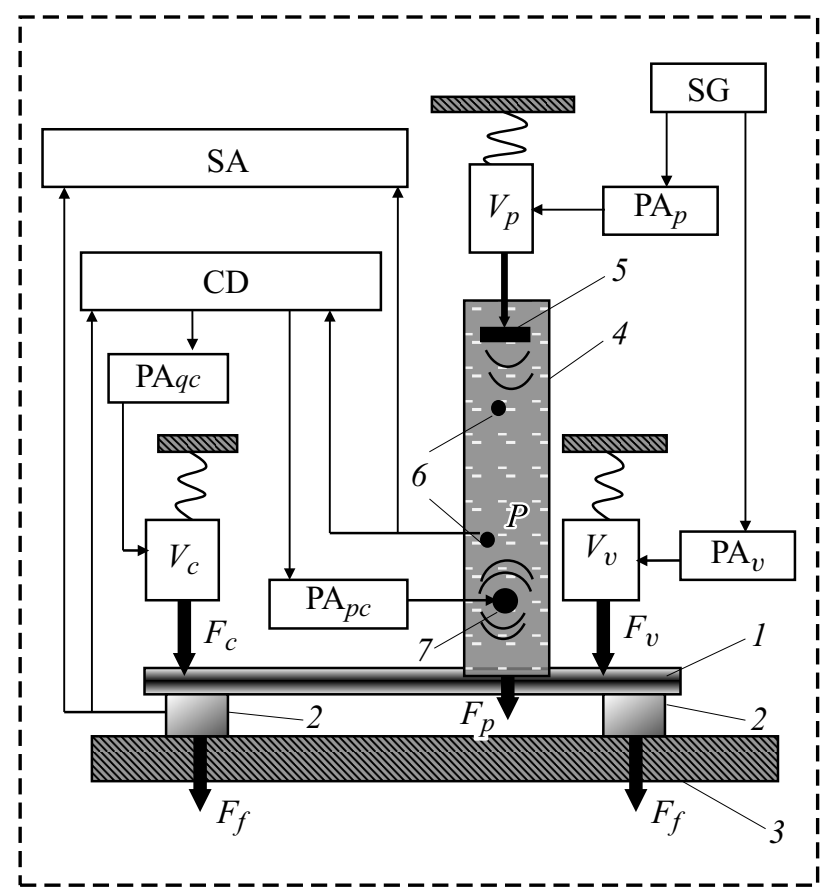

Рис. 1. Стенд опытной двухканальной $\mathrm{AC}$. $\mathrm{CD}-$ устройство управления (control device), РA - усилитель мощности (power amplifier), $V$ - вибратор (vibrator), SG - генератор сигналов (signal generator), SA - анализатор сигналов (signal analyzer). Индексы: $c-$ компенсация (compensation), $f-$ фундамент (fundament), $p$ - пульсации (pulsations), $v-$ вибрация (vibration), $q$ - сила (force). Двойной индекс $q c$ означает компенсацию силы (compensation of force) индекс $p c-$ компенсацию давления (compensation of pulsations). Остальные пояснения приведены в тексте.

Вибратор $V_{c}$ с усилителем мощности $\mathrm{PA}_{q c}$ создает компенсирующую силу $F_{c}$, которая действует на плиту $l$ и уменьшает измеряемую датчиком 2 силу $F_{f}$. Сигнал на $\mathrm{PA}_{q c}$ и $\mathrm{V}_{c}$ формируется в системе управления CD на основе обработки сигнала от датчиков силы $F_{f}$. Все вибраторы виброизолированы от плиты 1 и фундамента 3 при помощи упругого подвеса с собственной частотой менее $1 \mathrm{~Hz}$. Пьезокерамический излучатель 7 с усилителем мощности $\mathrm{PA}_{p c}$ создает пульсации

Письма в ЖТФ, 2018, том 44, вып. 24 
давления, уменьшающие пульсации, создаваемые поршнем 5. Сигнал на $\mathrm{PA}_{p c}$ и излучатель 7 формируется в системе управления $\mathrm{CD}$ на основе обработки сигнала от гидрофона 6.

В каналах компенсации силы и пульсации давления сигнал датчика обратной связи, датчика силы 2 или гидрофона 6 подается на один из входов аналого-цифрового преобразователя (АЦП) устройства управления CD. Сигнал оцифровывается и передается в процессорный модуль $\mathrm{CD}$, где с использованием цифрового регулятора формируется узкополосный или широкополосный компенсирующий сигнал. Сигнал подается на цифроаналоговый преобразователь (ЦАП), где преобразуется в аналоговый. Затем сигнал нормируется с помощью программируемых усилителей и подается на соответствующий усилитель мощности. Цифровое устройство управления содержит модуль питания, модуль АЦП, процессорный модуль на базе процессора STM32F7 с ядром Cortex-M7 с тактовой частотой $216 \mathrm{MHz}$, модуль ЦАП. Достижимые частоты дискретизации системы управления до $20 \mathrm{kHz}$. Регулятор в цепи обратной связи реализован в формате переменных пространства состояния. В качестве регуляторов на этом этапе работы использовались узкополосные и широкополосные фильтры: типа „резонансное звено“ (резонатор), эллиптические, Баттерворта и Чебышева различных порядков. Установка параметров устройства управления осуществляется от ЭВМ в среде MATLAB через последовательный интерфейс RS-485 на скорости $10 \mathrm{Mbit} / \mathrm{s}$. Контроль и обработка сигналов производились анализатором сигналов (SA) типа „Puls“ фирмы „Брюль и Къер“.

При проведении эксперимента для каждого из исследуемых вариантов подбирались характеристики регулятора (фильтра) и максимальный коэффициент усиления в цепи обратной связи для получения максимального гашения без потери устойчивости системы. Результаты активного гашения вибрационных сил $F_{f}$ под плитой представлены на рис. 2. На рис. 2, $a$ показано гашение шума при его возбуждении в полосе частот $0-400 \mathrm{~Hz}$ двумя резонаторами на частотах 110 и $194 \mathrm{~Hz}$. Уменьшение вибрационной силы в узкой полосе достигает почти порядка величины. На частотах ниже и выше полосы активного гашения возникают зоны отрицательной эффективности (рис. 2, $a-c$, $3, b)$. Они обусловлены тем, что регулятор (фильтр) на границах своей работы переворачивает фазу сигнала и вместо гашения усиливает исходный сигнал. Для повышения устойчивости и эффективности АС к широкополосным фильтрам добавлялись резонаторы. Они размещались на тех резонансных частотах, на которых наблюдался интенсивный

Письма в ЖТФ, 2018, том 44, вып. 24 

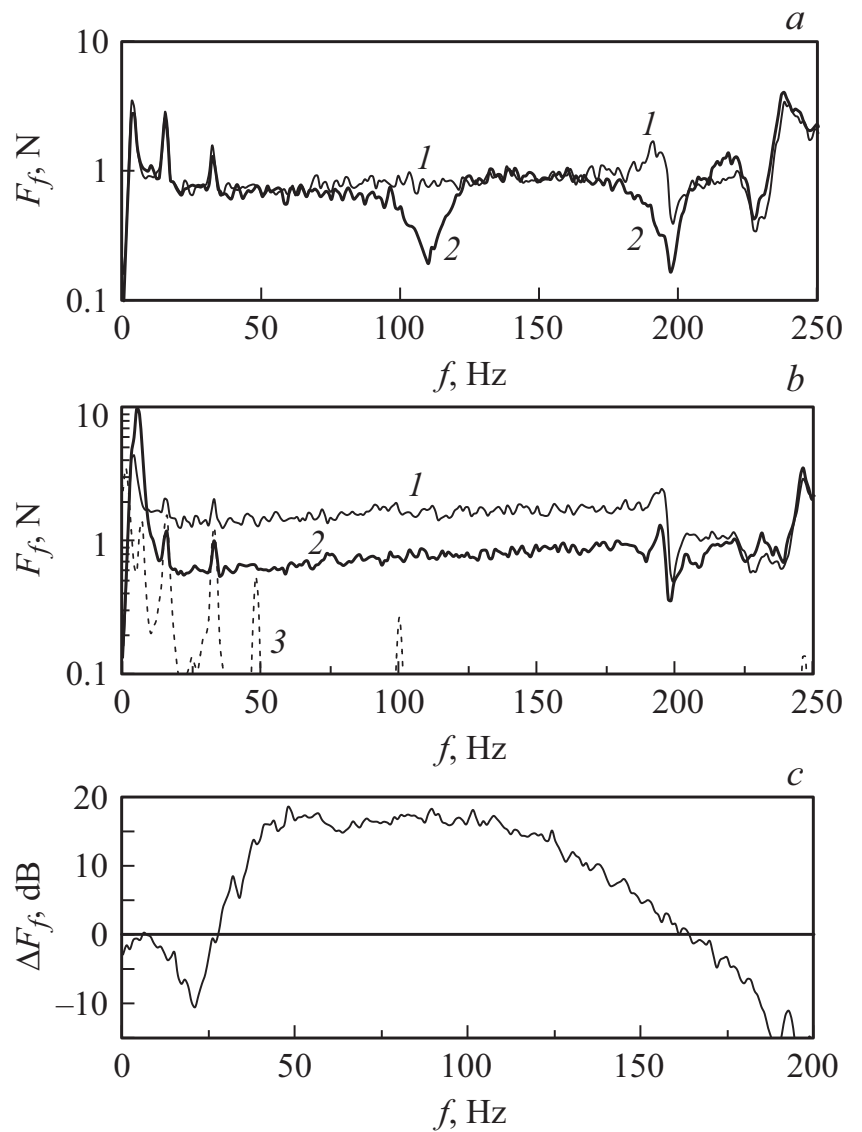

Рис. 2. Эффективность активного гашения вибрационных сил $F_{f}$ под плитой при возбуждении ее случайной силой с равномерным спектром в полосе частот. 1 - сигнал на контрольном датчике до гашения, $2-$ после гашения, 3 - помеха. $a-$ одновременное гашение на частотах 110 и $194 \mathrm{~Hz}$, фильтры типа „резонатор“; $b-$ эллиптический фильтр второго порядка в полосе $10-246 \mathrm{~Hz}$, резонаторы 190 и $246 \mathrm{~Hz} ; c$ - эффективность варианта с эллиптическим фильтром второго порядка (полоса частот $40-120 \mathrm{~Hz}$ ) и тремя резонаторами на частотах 165, 194 и $215 \mathrm{~Hz}$. 


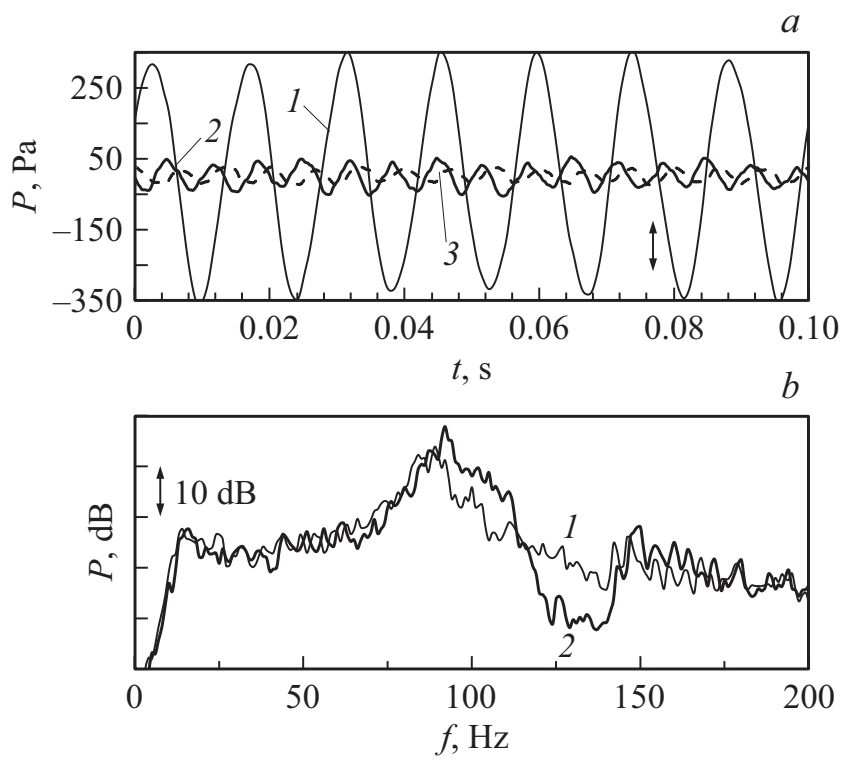

Рис. 3. Активное гашение пульсаций давления $P$ на дне трубы. $1-$ сигнал на контрольном гидрофоне до гашения, 2 - гашение, 3 - помеха. $a-$ синусоидальный сигнал (дискретная компонента) с частотой $70 \mathrm{~Hz} ; b-$ возбуждение равномерным спектром в полосе частот, эллиптический фильтр второго порядка в полосе $120-140 \mathrm{~Hz}$.

рост при увеличении коэффициента усиления в обратной связи при приближении к границе устойчивости. Резонаторы уменьшали уровни сил и повышали демпфирование в системе. На рис. $2, b$ приведены результаты активного гашения $F_{f}$ в полосе частот $10-246 \mathrm{~Hz}$ при помощи эллиптического фильтра второго порядка и двух резонаторов на частотах 190 и $246 \mathrm{~Hz}$, работающих совместно. Добавление двух резонаторов на частотах 190 и $246 \mathrm{~Hz}$ к эллиптическому фильтру позволило увеличить среднюю эффективность с $5 \mathrm{~dB}$ без резонаторов до $8 \mathrm{~dB}$ без потери устойчивости. Максимальная эффективность АС до $18 \mathrm{~dB}$ при гашении вибрационных сил под плитой была получена в полосе частот $40-120 \mathrm{~Hz}$ с эллиптическим фильтром второго порядка и тремя резонаторами (рис. 2,c). Применение фильтра Чебышева второго рода второго порядка с тремя резонаторами показало близкую эффектив-

Письма в ЖТФ, 2018, том 44, вып. 24 
ность. Варианты с фильтрами более высоких порядков оказались менее эффективными по сравнению с показанными. Как видно из сравнения pис. $2, b$ и $c$, при расширении полосы гашения от $40-120$ до $10-246 \mathrm{~Hz}$ при одинаковом типе регулятора, максимальная эффективность АС уменьшается с 18 до $8 \mathrm{~dB}$.

Осциллограммы пульсаций давления $P$ синусоидальной формы с частотой $70 \mathrm{~Hz}$ на дне трубы показаны на рис. $3, a$ до и после активного гашения. Исходный сигнал уменьшен на порядок. При возбуждении пульсаций давления случайным сигналом максимальное подавление при помощи резонатора составило порядок на частоте $120 \mathrm{~Hz}$. Активное гашение пульсаций в полосе частот $120-140 \mathrm{~Hz}$ эллиптическим фильтром второго порядка на рис. $3, b$ составило $10 \mathrm{~dB}$. Аналогично гашению вибрационной силы на частотах ниже и выше полосы гашения наблюдаются отрицательные эффекты.

Таким образом, можно сделать следующие выводы.

1. Исследован опытный образец активной системы. Он обеспечивает снижение вибрационных сил и пульсаций давления в трубопроводе до 10 раз как на отдельных дискретных частотах, так и в достаточно широких полосах частот в диапазоне от 10 до $246 \mathrm{~Hz}$.

2. Повышение эффективности АС при широкополосном гашении ограничено появлением автоколебаний в системе при увеличении коэффициентов усиления в цепи обратной связи. Это указывает на необходимость доработки алгоритма управления. Показано, что одним из способов может быть введение в него звеньев с демпфированием.

3. Дальнейшие исследования активного гашения вибрационных сил в компенсаторах трубопроводов должны быть направлены на исследование совместного гашения пульсаций и вибрационных сил и совершенствование алгоритмов управления.

Работа выполнена при поддержке Российского научного фонда (соглашение № 16-19-10292).

\section{Список литературы}

[1] Ганиев Р.Ф. Нелинейные резонансы и катастрофы. Надежность, безопасность, бесшумность. Москва: Dynamika, 2013. С. 505-542.

[2] Kiryukhin A.V., Milman O.O., Ptakhin A.V., Serezhkin L.N., Kondratev A.V. // Int. J. Appl. Eng. Res. 2017. V. 12. N 19. P. 8209-8216.

[3] Кирюхин А.В., Мильман О.О., Птахин А.В. // Теплоэнергетика. 2017. № 12. C. 58-66.

Письма в ЖТФ, 2018, том 44, вып. 24 